\title{
Hemolysis in medical devices
}

\begin{abstract}
Implantable assistive devices give hope of a permanent clinical solution to people with chronic failure, such as heart or kidney failures. While long-term use of continuousblood flow is being researched, the eliminating the extreme level of blood damage in these devices is an important design challenge. Blood damage or hemolysis depends on exposure time and shear stress, and device designers have usually preferred design estimations and calculations rather than experimental studies to make a decision in their design. In this mini review, the hemolysis aspects are reviewed to explain the risk of blood cell damage in implantable artificial devices.
\end{abstract}

Keywords: hemolysis prediction, red blood cells, shear stress, normalized index of hemolysis, cell damage, implantable devices
Volume 2 Issue 5 - 2017

\author{
Ali Ostadfar \\ Department of Pediatrics, University of British Columbia, \\ Canada \\ Correspondence: Ali Ostadfar, Department of Pediatrics, \\ University of British Columbia, Canada, \\ Email aostadfar@bcchr.ca
}

Received: March 20, 2017| Published: June 19, 2017

\section{Introduction}

Numerous types of assistive devices such as implantable or prosthesis devices have been developed and used for treating patients with chronic diseases. In an implantable device for instance an artificial kidney, the roughness of the surfaces, filter pores, and other components introduce shear stress in the system. This shear stress is well known as a factor causing hemolysis. ${ }^{1,2}$ The estimation of hemolysis inside an implantable artificial kidney is vital for the development of a blood-compatible device. Hemolysis is the damage of the red blood cells' membrane, and it is assessed by the amount of hemoglobin released from inside the red blood cells to the plasma. Hemolysis or damage of the red blood cells has been modeled with shear stress and exposure time (or accumulated number of events) in previous studies. ${ }^{3}$ Predicting or rate estimating in hemolysis issue is one of the best methods to analyze the problem without using physical experiments, and this method is a very valuable tool for medical devices which have direct contact with blood.

Various researches have examined the hemolysis in an implantable pump, and, while shear stress, ${ }^{4}$ solid friction, ${ }^{5}$ and heat generation ${ }^{6}$ have been listed as the main causes of hemolysis, it has also been suggested that roughness of surface has an influence. ${ }^{7}$ Umezu et al. ${ }^{8}$ reported an increase in the hemolysis level with a raise in the arithmetic mean level of roughness $(\mathrm{Ra})$, and they evaluated the detailed flow in the vicinity of the roughened surfaces. ${ }^{8}$

\section{Analysis and methods}

Outside the body, when RBCs are exposed to pressures up to 150 atmospheres, neither hemolysis nor a measurable change of osmotic fragility result. ${ }^{9}$ Recently, the hemolysis threshold was recognized at $130 \mathrm{MPa}(\sim 1300$ atmospheres $) .{ }^{10}$ Negative pressure also does not cause hemolysis. ${ }^{11}$ Only the dynamic element is related to blood damage caused by shear. Shear originates in the difference of fluid velocity between the blood layers in the channel; this difference creates a force which is called shear. Hemolysis is a nonlinear function of shear and exposure time $(t)$, and, regarding these relations, two equations are defined for hemolysis. The first equation (Eq.1) was introduced by Giersiepen et al. ${ }^{12}$

$$
\frac{\Delta H b}{H b}=3.62 \cdot 10^{-7} \cdot t^{0.785} \cdot \tau^{2.416}
$$

The second equation (Eq. 2) was proposed by Hauser et al. ${ }^{12}$

$$
\frac{\Delta H b}{H b} 1.8 \cdot 10^{-6} \cdot t^{0.765} \cdot \tau^{1.991}
$$

The Normalized Index of Hemolysis (NIH) is a standard clinical (experimental) indicator used to describe hemolysis in flow loop tests. The NIH is proportional to the increase of plasma free hemoglobin concentration, the volume of circulated blood, the volumetric flow rate of blood flow, and the hematocrit and time of the circulations. ${ }^{12}$

$$
\text { N.I.H. }\left(\frac{g}{100 L}\right)=\Delta H b . \mathrm{V} \cdot \frac{(100-\mathrm{Hct})}{100} \cdot \frac{100}{Q . t}
$$

Where $\Delta$ is the increase of plasma free hemoglobin concentration $(\mathrm{mg} / \mathrm{L}), \mathrm{V}$ is the volume of circulated blood $(\mathrm{L})$, is the blood hematocrit, $\mathrm{Q}$ is volumetric flow rate of the blood flow $(\mathrm{L} / \mathrm{min})$, and $t$ is the time of the test $(\mathrm{min})$. Surface roughness and the chemistry of the surface have major roles in hemolysis, and they may even govern capillary flow, especially at shear stress below the critical limit of $\sim 150 \mathrm{~Pa} .{ }^{13}$ Several hemolysis experiments conducted by researchers and investigators that all had similar tension for lysis, including but not limited to: Blachshear and Anderson (33dyne $/ \mathrm{cm}$ ), Rand (28.6dyne/cm) and Zydney $\left(25.6\right.$ dyne/cm). ${ }^{1}$ The studies and experiments demonstrate that the stress range for hemolysis is wide, between $150 \mathrm{~Pa}$ and $4000 \mathrm{~Pa}{ }^{1}$

Roughnesses exceeding $0.1 \mu \mathrm{m}$ appear to increase hemolysis rates significantly. ${ }^{14}$ Siliconizing (silicon coating) the internal surface of stainless steel tubing decreases hemolysis. ${ }^{15}$ Currently, this method is used for blood access cannulas in hemodialysis systems. In case of blood filtration, the cross-flow filtration method used to minimize cell deposition on the membrane surface also affects the hemolysis rate. The trans-membrane pressure drop, wall shear rate, and pore size are major factors that result in hemolysis in filtration membranes. These factors produce shear stress and membrane tension on red blood cell membranes which cause lysis.

\section{Conclusion}

Scale and type of stress on blood cell membrane have leading role in hemolysis. The stress will be affected by surface roughness and geometrical conditions of the device such as; the edges, the shape of 
the channel or pore inlets, also the flow regime of the fluid in bio-fluid separation. Most foci on hemolysis were on external assistive devices, recently, focus has turned to implantable heart pumps because these machines are operational in medical equipment, and researchers can make experiments using these machines. For other cases, such as an implantable artificial kidney, we have to use numerical or simulation methods to understand hemolysis aspects inside of these devices due to some limitations for real experiment in implantable devices. During procedure of device design, the hemolysis predicting can be calculated by using equation 1 to evaluate or predict cell damage in the system. The prediction results can help designers to change their design and compare the shear stress and other design parameters to the threshold of damage. This re-design and comparison is a useful tool in engineering design. As an alternative technique, finite element analysis methods and related software are very useful tools to understand the mechanical damage of blood cell membranes, ${ }^{16}$ without any doubt these tools have key role to reduce the hemolysis problems for artificial surfaces in both in vivo and in vitro conditions in future.

\section{Acknowledgements}

None.

\section{Conflict of interest}

The author declares no conflict of interest

\section{References}

1. Ostadfar A. Design and experimental proof of selected functions in implantable artificial kidney. Simon Fraser University; 2013. p. 1-156.

2. Yano T, Sekine K, Mitoh A, et al. An estimation method of hemolysis within an axial flowblood pump by computational fluid dynamics analysis. Artificial Organs. 2003;27(10):920-925.

3. Giersiepen M, Wurzinger LJ, Opitz R, et al. Estimation of shear stressrelated blood damage in heart valve prostheses in vitro comparison of 25 aortic valves. Int J Artif Organs. 1990;13(5):300-306.

4. Kameneva MV, Burgreen GW, Kono K, et al. Effects of turbulent stresses upon mechanical hemolysis: experimental and computational analysis. ASAIO J. 2004;50(5):418-423.
5. Orime Y, Shiono M, Yagi S, et al. Rota Flow RF-30 pump system: a new centrifugal blood pump for cardiopulmonary bypass. Artif Organs. 2000;24(6):437-441.

6. Göbel C, Arvand A, Eilers R, et al. Development of the MEDOS/ HIA DeltaStream extracorporeal rotary blood pump. Artif Organs. 2001;25(5):358-365.

7. Yasuda T, Shimokasa K, Funakubo A, et al. An investigation of blood flow behavior and hemolysis in artificial organs. ASAIO J. 2000;46(5):527531.

8. Yamazaki $\mathrm{K}$, Kormos $\mathrm{R}$, Mori $\mathrm{T}$, et al. An intraventricular axial flow blood pump integrated with a bearing purge system. ASAIO J. 1995;41(3):M327-M332.

9. Brewster E, Collins S, Funnell GR, et al. The effect of high pressure on the hemolysis of red blood cells. Under sea Biomed Res. 1976;3(2):151155 .

10. Yamaguchi T, Terada S, Ideguchi H. High-pressure-induced hemolysis of hereditary spherocytic erythrocytes is not suppressed by DIDS labeling. Jpn J Physiol. 1997;47(6):571-574.

11. Chambers SD, Laberteaux KR, Merz SI, et al. Effects of static pressure on red blood cells on removal of the air inter-face. ASAIO J. 1996;42(6):947-950.

12. A Ostadfar. Biofluid Mechanics: Principles and Applications. 1st ed. USA: Elsevier/Academic Press; 2016.

13. Polaschegg HD. Red blood cell damage from extracorporeal circulationin hemodialysis. Semin Dial. 2009;22(5):524-531.

14. Takami Y, Nakazawa T, Makinouchi K, et al. Effect of surface roughness on hemolysis in a pivot bearing supported Gyro centrifugal pump (C1E3). Artif Organs. 1996;20(11):1155-1161.

15. Blackshear PL, Dorman FD, Steichbach JH, et al. Shear, wall interactions and hemolysis. Trans Am Soc Artif Intern Organs. 1996;12:113-120.

16. Behr M, Arora D, Coronado O, et al. Models and Finite Element Techniques for Blood Flow Simulation. International Journal for Computational Fluid Dynamics; 2006. p. 1-12. 